\title{
CONCENTRACIÓN DE LEPTINA EN ADULTOS CON SOBREPESO SUJETOS A UN ENTRENAMIENTO CONCURRENTE
}

\section{LEPTIN CONCENTRATION IN OVERWEIGHT ADULTS UNDERGOING CONCURRENT TRAINING}

\author{
Rosa Guilherme ${ }^{1}$, Braga de Mello Danielli ${ }^{1,2}$, Daoud Rejane ${ }^{1}$, Cruz Iara $^{1}$ Dantas H. M. Estélio $^{1}$ \\ 1 - Laboratório de Biociências da Motricidade Humana - Universidade Federal do Estado do Rio de Janeiro - \\ LABIMH/UNIRIO - RJ/Brasil. \\ 2 - Escola de Educação Física do Exército - EsEFEx/EB/RJ/Brasil.
} ROSA G., BRAGA de MELLO D., DAOUD R., CRUZ I. \& DANTAS H.M.E. Concentración de Leptina en adultos con
sobrepeso sujetos a un entrenamiento concurrente. Mot. Hum., 10(2): 95-102, 2010.

\section{RESUMEN}

El objetivo es Investigar los efectos de diferentes intensidades de entrenamiento concurrente (EC) sobre los niveles de leptina en adultos con sobrepeso que practican ejercicio regularmente. Treinta sujetos $(27,1 \pm 4,8$ años) fueron asignados en: grupo control $(G C)$, el entrenamiento concurrente $A(E C A)$ y entrenamiento concurrente $B$ (ECB). Se recogieron muestras de sangre para la leptina. El EC se ha caracterizado por ciclismo indoor seguido por el entrenamiento con pesas. En el grupo de ECA ciclismo indoor duró 40 min, con una intensidad en una escala de 5-7 OMNI y el trabajo de pesas se realizó con una intensidad del 85\% de 1RM y con intervalos de 2-3 minutos entre series. En el ECB en el ciclismo indoor tenían las mismas características de la ECA, sin embargo, el entrenamiento de fuerza se realizó con una intensidad del $60 \%$ de 1 RM y los intervalos de 1 minuto entre series. El GC no realizo ejercicios físicos. Al final, se llevó a cabo en los grupos una nueva extracción de sangre. Se utilizó la prueba de Shapiro-Wilk, y ANOVA de dos vías, con comparaciones Post-Hoc según Tukey. Una reducción significativa en los niveles de leptina después de ECA $(\Delta=-16,04 \%, p=0,05)$ y ECB $(\Delta=-$ 10,70\%, p = 0,46). Conclusión: El EC causó una reducción en los niveles de leptina, independientemente de la intensidad aplicada, sin embargo, sólo la formación con las características de alta intensidad causó una reducción significativa en los niveles de la variable.

Palabras clave: leptina, ejercicio, hormonas.

\section{INTRODUCCION}

Entre las sustancias producidas por el tejido adiposo se encuentra la leptina (1), compuesta de 167 aminoácidos del péptido encontrado principalmente por el tejido adiposo blanco, sin embargo, su presencia se observa también en otros tejidos como el estómago, la glándula mamaria y la placenta $(2,3)$.

Su nombre se deriva de la palabra "leptos" que en griego significa "flaco" (4), y es considerado como el controlador del peso corporal debido al hecho de que transmite información al hipotálamo sobre la cantidad de energía almacenada en el tejido adiposo, suprimiendo el apetito y afectando el gasto de energía (5).

Además de su función endocrina y metabólica, estudios recientes $(6,8)$ también clasifican la leptina como una hormona que interviene en la respuesta inflamatoria, dada su clara interacción con el sistema inmunológico. Parece tener también la participación en el control de los sistemas hematopoyético, reproductivo, cardiovascular y el metabolismo óseo (9).

Los efectos del ejercicio sobre los niveles de leptina se han investigado en estudios previos $(10,13)$ después de procedimientos aeróbicos o de la fuerza de forma aislada. Sin embargo, poco se sabe sobre los efectos del entrenamiento concurrente sobre los niveles séricos de la leptina. El entrenamiento concurrente es una estrategia que combina el ejercicio aeróbico y entrenamiento de la fuerza en el mismo período de sesiones $(14,15)$. Es ampliamente utilizado ya que los beneficios del entrenamiento de 
ROSA G., BRAGA de MELLO D., DAOUD R., CRUZ I. \& DANTAS H.M.E. Concentración de Leptina en adultos con sobrepeso sujetos a un entrenamiento concurrente. Mot. Hum., 10(2): 95-102, 2010.

fuerza y resistencia aeróbica son adquiridos al mismo tiempo (16).

Aparte de la modalidad a realizar, la intensidad del ejercicio se presenta como otro factor en el comportamiento de la leptina 17,18). Por lo tanto, el propósito de este estudio fue investigar los efectos de dos protocolos de entrenamiento que compiten en diferentes intensidades en los niveles de leptina en adultos con sobrepeso que participan en el ejercicio regular.

\section{MATERIAL Y MÉTODOS}

Esta investigación es experimental, ya que trata de establecer relaciones de causa y efecto entre las variables investigadas (20)

Fue utilizada una muestra de 30 voluntarios masculinos, que practican regularmente ejercicio físico durante al menos seis meses en la Academia WestFit, localizada en la ciudad de Río de Janeiro (Brasil) con una frecuencia semanal de al menos tres días y el factor de riesgo evidente que podrían impedir su participación en el estudio de acuerdo a los criterios de estratificación del riesgo de la Asociación Americana del Corazón (21).

Los sujetos fueron asignados de forma aleatoria por simple sorteo en tres grupos iguales: control (GC), que no ha practicado ningún tipo de ejercicio físico, el entrenamiento concurrente A (ECA), que llevó a cabo la sesión de entrenamiento de alta intensidad, y concurrente de formación B (ECB), que llevó a cabo la sesión de entrenamiento con una intensidad moderada.

Los sujetos firmaron un consentimiento informado para participar en la investigación con seres humanos, de conformidad con las normas de la Declaración de Helsinki (22) y la Resolución 196/96 del Consejo Nacional de Salud, 10/10/1996. El estudio tuvo su proyecto de investigación presentado y aprobado (con el $\mathrm{N}^{\circ}$ 0189/2008) por la Ética en Investigación con Seres Humanos de la Universidad Castelo Branco - UCB / RJ.

En la primera etapa, los sujetos fueron respondiendo a los criterios para la estratificación del riesgo de la American Heart Association (AHA) en los criterios de estratificación de riesgo para el ejercicio físico
(21). Además, se tomaron medidas de la masa corporal total y la altura determinado el índice de masa corporal (IMC) para las características antropométricas de la muestra.

Para la evaluación de la masa corporal total y la altura, se utilizó una escala de capacidad mecánica de $150 \mathrm{~kg}$ y precisión $100 \mathrm{~g}$ con estadiómetro de la firma Filizola $₫$ (Brasil). Los procedimientos adoptados establecidos por la Sociedad Internacional para la Promoción de la Cineantropometría - ISAK (23). Bajo índice de masa corporal se obtuvo por la relación de la masa corporal en kilogramos y la altura en metros al cuadrado (24) y se utilizó para la clasificación de la OMS (25).

En la segunda etapa, los sujetos fueron evaluados utilizando la repetición máxima (1RM) (26) en los ejercicios de apoyo de Remada, $45^{\circ}$ prensa de piernas, press de banca, extensión de la rodilla (extensión de la pierna), la flexión del codo (HBM), flexión Rodilla (enrollamiento de la pierna) y extensión del codo (polea alta), que permite medir la carga máxima y el cálculo posterior de la intensidad del entrenamiento.

En la etapa siguiente, los sujetos realizaron una clase de ciclismo indoor con la escala de esfuerzo percibido OMNI Para Ciclismo propuesto por Robertson (27).

\section{Grupo de Control:}

Se recogieran muestras de sangre después de ayunar durante 12 horas, con el objetivo de verificar los niveles de referencia de la leptina. Ninguno de los participantes hizo ningún tipo de actividad física el día antes de la sesión.

Las muestras de sangre fueron recolectadas en el sitio de la intervención por el equipo de técnicos calificados en la medicina de diagnóstico de laboratorio Sergio Franco - Brasil, y se analizaran por el método de radioinmunoensayo para la medición de la leptina en suero, utilizando el Leptin Humanos Ria kit (Linco Research, Inc., St. Louis, MO, EE.UU.).

Después de eso, los sujetos realizaron el desayuno que consiste en $200 \mathrm{ml}$ de yogur $0 \%$ grasa, dos 
ROSA G., BRAGA de MELLO D., DAOUD R., CRUZ I. \& DANTAS H.M.E. Concentración de Leptina en adultos con sobrepeso sujetos a un entrenamiento concurrente. Mot. Hum., 10(2): 95-102, 2010.

rebanadas de pan de trigo, $30 \mathrm{~g}$ de queso fresco, margarina vegetal $10 \mathrm{~g}$ y un plátano mediano. Dos horas después de la primera reunión se realizo una nueva colecta de muestras de sangre. Estos procedimientos eran las 6:30 am y $8: 30$ pm respectivamente.

Grupo ECA:
Para este grupo, las muestras de sangre fueron tomadas siguiendo el modelo adoptado en el grupo control. Cuarenta minutos después del desayuno, el grupo realizo la sesión de entrenamiento : Clase de ciclismo indoor con el método continuo, que dura unos 40 minutos, y la intensidad de entre cinco y siete de la escala OMNI de esfuerzo percibido para el ciclismo (27), como se muestra en la Tabla I.

\begin{tabular}{ccc}
\hline Tiempo $(\mathbf{m i n})$ & Fase & Intensidad (OMNI) \\
\hline $\mathbf{1 - 5}$ & Calentamiento & $2-4$ \\
$\mathbf{5 - 3 5}$ & Entrenamiento contínuo & $5-7$ \\
$\mathbf{3 5 - 4 0}$ & Vuelta à la calma & $0-2$ \\
\hline
\end{tabular}

Tabla I. Protocolo de Ciclismo Indoor

Después se realizó una sesión de entrenamiento con pesas que se compone de tres series de repeticiones realizadas hasta el agotamiento para cada ejercicio probado, dispuestos en las series presentadas en la Tabla II.

La intensidad fue de $85 \%$ de 1RM para todos los ejercicios y el descanso entre series fue de 2 a 3 min.

\begin{tabular}{lcc}
\hline \multicolumn{1}{c}{ Ejercicio } & Series & Rep. \\
\hline Remada Apoyada & 3 & agotamiento \\
Leg Press $\mathbf{4 5}^{\circ}$ & 3 & agotamiento \\
Supino Reto & 3 & agotamiento \\
Extensión de rodilla & 3 & agotamiento \\
Flexión de Codos & 3 & agotamiento \\
Flexión de rodilla & 3 & agotamiento \\
Extensión Codos (Polea) & 3 & agotamiento \\
\hline
\end{tabular}

Tabla II. Protocolo de Pesas

Después de estos procedimientos, los sujetos se sometieron a una nueva extracción de sangre.

\section{Grupo ECB:}

En este grupo, se aplicaron los mismos procedimientos sin embargo, la intensidad del entrenamiento fue modificada. En primer lugar, los sujetos realizaron clases de ciclismo indoor con características similares de duración y de la intensidad del grupo de ECA. Después de eso, hubo una sesión de entrenamiento de pesas siguiendo la misma serie se muestra en la Tabla 2, sin embargo, este grupo se utilizó la intensidad del $60 \%$ de 1 RM con un intervalo de 1 minuto entre series.
La intervención se produjo en la misma fecha para todos los grupos. Durante la reunión, los sujetos de cada grupo consumió sólo agua ad libitum.

Todos los procedimientos estadísticos fueron procesados utilizando el programa Statistical Package for the Social Sciences (SPSS 14.0, Chicago, EE.UU.). Se utilizaron estadísticas descriptivas para presentar los valores de las medidas de tendencia central y dispersión. Para analizar la normalidad de los datos se utilizó la prueba de Shapiro-Wilk y el análisis de la diferencia observada entre los grupos se utilizó para ANOVA de dos vias con comparaciones y PostHoc de Tukey. El nivel de significación fue de $95 \%$ (p <0,05). 
ROSA G., BRAGA de MELLO D., DAOUD R., CRUZ I. \& DANTAS H.M.E. Concentración de Leptina en adultos con sobrepeso sujetos a un entrenamiento concurrente. Mot. Hum., 10(2): 95-102, 2010.

\section{RESULTADOS}

La Tabla III presenta datos sobre las características antropométricas y de normalidad de los individuos de los grupos que componen el estudio.
La Tabla IV presenta los resultados sobre el comportamiento de las concentraciones de leptina en cada grupo que escribió el estudio.

\begin{tabular}{cccc}
\hline & Masa Corporal $(\mathbf{K g})$ & Estatura(m) & IMC \\
\hline GC & $79,24 \pm 7,10$ & $1,71 \pm 0,02$ & $27,07 \pm 2,65$ \\
SW (p-valor) & 0,13 & 0,57 & 0,79 \\
ECA & $78,72 \pm 6,67$ & $1,70 \pm 0,02$ & $27,23 \pm 2,50$ \\
SW (p-valor) & 0,39 & 0,51 & 0,27 \\
ECB & $76,92 \pm 5,67$ & $1,68 \pm 0,14$ & $27,25 \pm 1,51$ \\
SW (p-valor) & 0,22 & 0,54 & 0,38 \\
\hline
\end{tabular}

Tabla III. Características antropométricas de los indivíduos.

Leyenda: GC: grupo control; ECA: Grupo entrenamiento concurrente A; ECB: Grupo entrenamiento concurrente B, IMC: índice de masa corporal; SW: Shapiro-Wilk.

\begin{tabular}{cccc}
\hline Leptina (mg/dl) & GC & ECA & ECB \\
\hline PRÉ & $5,12 \pm 1,01$ & $9,97 \pm 6,12$ & $15,69 \pm 6,77$ \\
PÓS & $4,90 \pm 0,83$ & $8,37 \pm 4,99$ & $14,01 \pm 8,46$ \\
$\Delta \%$ & $-4,29$ & $-16,04$ & $-10,70$ \\
p-valor & 0,11 & $0,05^{*}$ & 0,46 \\
\hline
\end{tabular}

Tabla IV. Niveles de leptina antes y después de diferentes intervenciones.

Leyenda: GC: grupo control; ECA: Grupo entrenamiento concurrente A; ECB: Grupo de Entrenamiento concurrente B * Diferencia significativa $(\mathrm{p}<0,05)$ dentro de los grupos.

Se observa que la reducción en los niveles de leptina en todos los grupos, sin embargo, sólo el grupo TCA mostraron una reducción estadísticamente significativa $(\mathrm{p}<0,05)$.

\section{DISCUSIÓN}

Para Benatti et al. (1), el desequilibrio de la energía inducida por el esfuerzo físico parece ser esencial para posibles cambios agudos en las concentraciones de leptina. Hubo una reducción en los niveles de leptina en todos los grupos que componían el presente estudio, el cual es estadísticamente significativo sólo en el grupo que llevó a cabo el entrenamiento con características de alta intensidad.
Los resultados de este estudio son coincidentes con estudios de Rosa et al (28), Eguchi et al (29) y Negrão (30), que relatan que el ejercicio físico tiene la capacidad de influir en los niveles de leptina.

Para Hulver (17), sesiones de ejercicios de alta intensidad y de duración superior a una hora parecen ser más eficaz en la reducción en las concentraciones de leptina. Según Meira et al. (18), cuando más alto es el nivel de estrés causado por el ejercicio físico, menor será la concentración de leptina encontrada. Estos datos son coincidentes con los resultados de este estudio, en los que se produjo una reducción significativa en los niveles de leptina en el grupo que realizó el protocolo de ejercicio con las características de mayor intensidad. 
ROSA G., BRAGA de MELLO D., DAOUD R., CRUZ I. \& DANTAS H.M.E. Concentración de Leptina en adultos con sobrepeso sujetos a un entrenamiento concurrente. Mot. Hum., 10(2): 95-102, 2010.

En su investigación, Zafeiridis et al. (19) también se observa la reducción en los niveles de leptina después de protocolo de ejercicio con una intensidad similar a la utilizada por el grupo de ECA en este estudio. Además, su trabajo muestra que, como en el presente estudio, el grupo que realizó el protocolo de ejercicio con la intensidad similar a la del grupo ECB no mostró diferencias significativas en los niveles de leptina.

Jürimäe (10) y Keller (13) también relataron una disminución en los niveles de leptina después de una sola sesión de ejercicio, como en este estudio. Sin embargo, los resultados de la investigación de Eguchi et al. (29) son contradictorias con estos, ya que se observó un aumento de los niveles de leptina después de su protocolo de intervención.

Weltman et al. (31) encontraron que el ejercicio físico a intensidades diferentes, no afectó los niveles de leptina en los sujetos de su muestra. Lo mismo ocurrió en el estudio de Zoladz et al. (32). Por el contrario, como en este estudio, Kanaley (11) encontraron niveles bajos de leptina en la fase aguda del programa de entrenamiento utilizado en su intervención.

En su investigación, Rosa et al. (28), utilizaron una sesión de protocolo de intervención de entrenamiento concurrente con características similares a lo ECA de este estudio en cuanto a las modalidades utilizadas, el número de ejercicios, el orden de ejecución, la intensidad, y el intervalo de tiempo. Sus resultados muestran que, como en el presente estudio, una sesión de entrenamiento fue capaz de inducir una reducción significativa en los niveles de leptina.

Estadella et al. (33) observó en su estudio que la disminución de los niveles de leptina fue acompañada por cantidades pequeñas de peso corporal y de la masa grasa. Estos resultados destacan la importancia del ejercicio físico sobre el control de los niveles de leptina y el peso corporal, por lo que la pérdida de peso se puede lograr.

\section{CONCLUSIÓN}

Se puede concluir que el protocolo de entrenamiento concurrente de alta intensidad causó una reducción en los niveles plasmáticos de leptina en adultos con sobrepeso que practican ejercicios físicos regularmente. Por lo tanto, el entrenamiento concurrente realizado a una intensidad alta se presenta como una alternativa para reducir los niveles de leptina y puede actuar directamente en la pérdida de peso, el control del apetito y la masa corporal.

Estudios recomiendan nuevas investigaciones que relacionen el entrenamiento concurrente con los niveles séricos de leptina, así como el comportamiento de esta variable en respuesta a otras modalidades del ejercicio físico.

\section{REFERENCIAS BIBLIOGRAFICAS}

1. Benatti FB. Leptina e exercício físico aeróbio: implicações da adiposidade corporal e insulina. Rev Bras Med Esporte 2007;13(4):263-9.

2. Blüher S, Mantzoros C. Leptin in humans: lessons from translational research. Am J Clin Nutr 2009;89:991-997.

3. Ribeiro S, Santos Z, Silva RWd, Louzada E, Donato Júnior J, Tirapegui J. Leptina: Aspectos Sobre o Balanço Energético, Exercício Físico e Amenorréia do Esforço. Arq Bras Endocrinol Metab 2007;51(1):11-24.

4. Sanchez J. Perfil fisiológico de la leptina. Colombia Médica 2005;36(1):50-59.

5. Souza M, Brás-Silva C, Leite-Moreira A. O papel da leptina na regulação da homeostasia energética. Acta Med Port 2009;22:291-298.

6. Zulet M, Puchau B, Navarro C, Martí A, Martínez Hernández J. Biomarcadores del estado inflamatorio: nexo de unión con la obesidad y complicaciones asociadas. Nutrición Hospitalaria 2007;22(5):511527.

7. Iikuni N, Lam QLK, Lu L, Matarese G, Cava AL. Leptin and Inflammation. Curr Immunol Rev 2008;4(2):70-79.

8. Conde J, Scotece M, Gómez R, Gómez-Reino JJ, Lago F, Gualillo O. At the crossroad between immunity and metabolism: focus on leptin. Expert Rev Clin Immunol 2010;6(5):801-818. 
ROSA G., BRAGA de MELLO D., DAOUD R., CRUZ I. \& DANTAS H.M.E. Concentración de Leptina en adultos con sobrepeso sujetos a un entrenamiento concurrente. Mot. Hum., 10(2): 95-102, 2010.

9. Mota GR, Zanesco A. Leptina, Ghrelina e Exercício Físico. Arq Bras Endocrinol Metab 2007;51(1):25-33.

10. Jürimäe J. Leptin responses to short term exercise in college level male rowers. Br J Sports Med 2005;39(1):6-9.

11. Kanaley JA. Resting leptin responses to acute and chronic resistance training in type 2 diabetic men and women. Int $\mathrm{J}$ Obes Relat Metab Disord 2001;25(10):1474-1480.

12. Landt M. Prolonged exercise decreases serum leptin concentrations. Metabolism 1997;46(10):1109-1112.

13. Keller P. Leptin gene expression and systemic levels in healthy men: effect of exercise, carbohydrate, interleukin-6, and epinephrine. J Appl Physiol 2005;98:1805-1812.

14. Bell GJ. Effect of concurrent strength and endurance training on skeletal muscle properties and hormone concentrations in humans. Eur J Appl Physiol 2000;81:418-427.

15. Dantas EHM. Effects of a programme for years enderers physical force on the muscle and body composition of adults. Sport Sci Health 2008;4:1519.

16. Leveritt M. Concurrent strength and endurance training: the influence of dependent variable selection. Journal of Strength and Conditioning Research 2003;17(3):503-508.

17. Hulver MW. Plasma leptin and exercise: recent findings. Sports Med2003;33(7):473-482.

18. Meira TdB, Moraes FLd, Böhme MTS. Relações entre leptina, puberdade e exercício no sexo feminino. Revista Brasileira de Medicina do Esporte2009;15:306-10.

19. Zafeiridis A, Smilios I, Considine R, Tokmakidis $\mathrm{S}$. Serum leptin responses after acute resistance exercise protocols. J Appl Physiol2003;94:591-597.
20. Thomas Jr, Nelson Jk, Silverman Sj. Métodos de pesquisa em atividade física. 5 ed: Artmed Editora; 2007.

21. ACSM's Guidelines For Exercise Testing And Prescription. 7th ed: Lippincott Williams \& Wilkins; 2006.

22. W.M.A. Declaration Of Helsinki. Ethical Principles for Medical Research Involving Human Subjects. 59th WMA General Assembly, Seoul2008.

23. Marfell-Jones M. International standards for anthropometric assessment. ISAK: Potchefsroom, South Africa 2006.

24. Nihiser AJ, Lee SM, Wechsler H, McKenna M, Odom E, Reinold C, et al. BMI Measurement in Schools. Pediatrics 2009;124:89-97.

25. W.H.O. Obesity: preventing and managing the global epidemic. Report of a WHO Consultation. WHO Technical Report Series 894: World Health Organization; 2000.

26. Baechle TR, Earle RW. Essencials of strength training and conditioning: Champaign: human kinetics; 2000.

27. Robertson RJ. Validation of the Adult OMINI Scale of Perceived Exertion for Cycle Ergometer Exercise. Med Sci Sports Exerc 2004;36(1):102-108.

28. Rosa G, Cruz I, Mello DBd, Fortes MdSR, Dantas EHM. Plasma levels of leptin in overweight adults undergoing concurrent training International SportMed Journal 2010;11(3).

29. Eguchi R, Cheik NC, Oyama LM, Nascimento CMOd, Mello MTd, Tufik S, et al. Efeitos do exercício crônico sobre a concentração circulante da leptina e grelina em ratos com obesidade induzida por dieta. Rev Bras Med Esporte 2008;14:182-187.

30. Negrão $\mathrm{AB}$, Licinio J. Leptina: o Diálogo entre Adipócitos e Neurônios. Arq Bras Endocrinol Metab 2000;44(3):205-214.

31. Weltman A, Pritzlaff C, Wideman L, Considine R, Fryburg D, Gutgesell M. Intensity of acute exercise does not affect serum leptin concentrations 
ROSA G., BRAGA de MELLO D., DAOUD R., CRUZ I. \& DANTAS H.M.E. Concentración de Leptina en adultos con sobrepeso sujetos a un entrenamiento concurrente. Mot. Hum., 10(2): 95-102, 2010.

in young men. Med Sci Sports Exerc 2000;32(9):1556-1561.

32. Zoladz J, Konturek S, Duda K, Majerczak J, Sliwowski Z, Grandys M, et al. Effect of modarate incremental exercise, performed in fed and fasted state on cardio-respiratory variables and leptin and ghrelin concentrations in young healthy men. Journal Of Physiology And Pharmacology 2005;53(1):63-85.

33. Estadella D, Oyama L, Dâmaso A, Ribeiro E, Nascimento C. Effect of palatable hyperlipidic diet on lipid metabolism of sedentary and exercised rats. Nutrition 2004;20:218-224. 
ROSA G., BRAGA de MELLO D., DAOUD R., CRUZ I. \& DANTAS H.M.E. Concentración de Leptina en adultos con sobrepeso sujetos a un entrenamiento concurrente. Mot. Hum., 10(2): 95-102, 2010.

\begin{abstract}
To investigate the effects of different intensities of concurrent training (CT) on leptin levels in overweight adults engaged in physical exercise. Thirty subjects $(27.1 \pm 4.8$ years) were randomized into control group $(C G)$, concurrent training A (CTA) and concurrent training B (CTB). Blood samples of leptin were collected. The CT was characterized by indoor cycling followed by weight training. In the CTA group indoor cycling lasted for 40 min, with intensity between 5-7 of the OMNI scale and strength training was performed with an intensity of 85\% IRM and intervals of 2-3 minutes between sets. In CTB indoor cycling had the same characteristics of the CTA, however, strength training was performed with an intensity of 60\% of 1 RM and 1-minute intervals between sets. The CG did not perform physical exercises. At the end, new blood sample were assessed in the groups. Shapiro-Wilk test, Two-Way ANOVA and Tukey Post-Hoc test were used. There was a significant reduction on leptin levels after CTA $(\Delta \%=-16,04 ; p=0,05)$ and CTB $(\Delta \%=-10,70 ; p$ =0,46). CT promoted reduction in leptin levels independently of the intensity applied, however, just the training with elevated overload characteristics induced significant reduction on variable levels.
\end{abstract}

Key Words: Leptine, physical exercise, hormones.

Dirigir correspondencia a:

Guilherme Rosa

Dirección: Rua Piraquara, 879. Realengo. Rio de Janeiro

BRASIL.

CEP: 21755-270

Teléfono: $55212401-5243$

e-mail: grfitness@hotmail.com

RECIBIDO 6-12-2010

ACEPTADO 24-12-2010 